\title{
The efficacy of cisplatin on nasopharyngeal carcinoma cells may be increased via the downregulation of fibroblast growth factor receptor 2
}

\author{
LI PU ${ }^{1}$, LIZHONG SU ${ }^{1}$ and XIXUN KANG ${ }^{2}$ \\ ${ }^{1}$ Department of Otolaryngology, Zhejiang Provincial People's Hospital, People's Hospital of Hangzhou Medical College, \\ Hangzhou, Zhejiang 310014; ${ }^{2}$ Department of Otolaryngology, Head and Neck Surgery, Shenzhen Hospital, \\ University of Chinese Academy of Sciences, Shenzhen, Guangdong 518106, P.R. China
}

Received October 19, 2018; Accepted April 12, 2019

DOI: 10.3892/ijmm.2019.4193

\begin{abstract}
Cisplatin is one of the primary compounds used in the treatment of nasopharyngeal carcinoma (NPC), and fibroblast growth factor receptor 2 (FGFR2) has emerged to be a promising target for treatment in various tumors. Therefore, the present study aimed to explore whether the expression levels of FGFR2 in NPC tissues and cell lines were altered, and whether the efficiency of cisplatin was increased following the downregulation of FGFR2. The downregulation of FGFR2 was achieved by transfection with a small interfering RNA against FGFR2. Tissues of patients with NPC were analyzed by immunohistochemistry. Cell viability was examined using a Cell Counting Kit-8 assay. Cell cycle analysis was performed using flow cytometry. mRNA and protein levels were measured by reverse transcription quantitative polymerase chain reaction and western blot analysis, respectively. FGFR2 was observed to be overexpressed in cancer tissues of patients with NPC and in the NPC SUNE1, C666-1, 6-10B and HNE-3 cell lines, and resulted in an unfavorable prognosis. Cisplatin treatment decreased cell viability and increased FGFR 2 expression. The silencing of FGFR2 was demonstrated to augment the effects of cisplatin treatment, including decreasing the cell viability and inducing cell cycle arrest, which involved the increase and decrease of the durations of G1 and S phases, respectively, and a decrease in the expression levels of cyclin D1 and CDC25A, and increasing the rate of apoptosis via the intrinsic apoptosis pathway, as demonstrated by the upregulation of cleaved caspase- 3 and B-cell lymphoma 2 (Bcl-2)-associated X protein and downregulation of Bcl-2, in SUNE1 and C666-1 cell lines.
\end{abstract}

Correspondence to: Dr Xixun Kang, Department of Otolaryngology, Head and Neck Surgery, Shenzhen Hospital, University of Chinese Academy of Sciences, 4253 Songbai Road, Guangming, Shenzhen, Guangdong 518106, P.R. China

E-mail: xixunk_kangxx@163.com

Key words: cisplatin, nasopharyngeal carcinoma, fibroblast growth factor receptor 2, efficiency, mechanism
FGFR2 was overexpressed in the cancer tissues of patients with NPC and in NPC cell lines, resulting in an unfavorable prognosis. The downregulation of FGFR2 decreased cell viability via cell cycle arrest at G1 phase, and increased the efficacy of the cisplatin-based induction of apoptosis through the intrinsic apoptosis pathway.

\section{Introduction}

Nasopharyngeal carcinoma (NPC) is a common malignant tumor of the head and neck, with a high incidence in South Asia and Southern China (1). At present, the primary treatment methods of NPC are radiotherapy and chemotherapy, which have clinically-demonstrated antitumor effects; however, the 5 -year overall survival rate of patients with NPC remains low $(1,2)$. The mechanism of NPC has not yet been elucidated, although an accelerated cell cycle and enhanced cell invasion are considered to be closely associated with the occurrence and development of NPC (3). A variety of genes are involved in the regulation of cell proliferation and invasion in NPC (3). However, appropriate target genes for NPC treatment have not been identified.

Fibroblast growth factor receptors (FGFRs) belong to a family of transmembrane tyrosine kinase receptors with autophosphorylation activity (4). A total of 4 family members, FGFR1, FGFR2, FGFR3 and FGFR4, have been identified (4). Overexpression, mutations and abnormal transcriptional regulation of FGFRs may cause abnormalities in the FGFR downstream signaling pathway, leading to tumor formation (5). The FGFR downstream signaling pathway has been identified to serve a critical role in the development of prostate and skin cancer, transitional cell carcinoma and hematological malignancies (5). FGFR2 is a product of the expression of the oncogene BEK, which is located on chromosome 10 (10q26), and induces dimerization of FGFR2, autophosphorylation of intracellular kinases and conformational changes of the receptor by binding to fibroblast growth factors (FGFs). In addition, it has been demonstrated to cause the activation of a series of downstream cascade signaling pathways, including the mitogen-activated protein kinase/extracellular signal-regulated kinase and phosphatidylinositol 3-kinase/RAC-alpha 
serine/threonine-protein kinase pathways (6,7). Overexpression and missense mutations of the FGFR2 gene have been identified in a variety of human tumors, including gastric, breast and ovarian cancer (8-10). However, to the best of our knowledge, there have been few studies investigating FGFR2 in NPC.

Cisplatin has been widely used in the treatment of a variety of tumors following approval by the United States of America Food and Drug Administration in 1978 (11). Patients usually experience a good therapeutic effect in the early stages of cisplatin chemotherapy; however, it is common for drug resistance to develop gradually during treatment and severely limit the efficacy of subsequent cisplatin therapy (12). In addition, certain patients have been observed to exhibit intrinsic resistance to cisplatin (12). Cisplatin resistance has also been identified in NPC cells, and is becoming a serious public health concern $(13,14)$.

In light of these data, we hypothesized that FGFR2 served a critical role in the effect of cisplatin on the viability and apoptosis of NPC cells. Therefore, the present study aimed to explore whether the expression levels of FGFR2 in NPC tissues and cell lines were altered, and whether the efficiency of cisplatin was improved following the downregulation of FGFR2, in order to reveal the potential of FGFR2 in improving the efficacy of cisplatin treatment.

\section{Materials and methods}

Collection of cancer and adjacent tissues from NPC patients. The study protocol was approved by the Ethics Board of Zhejiang Provincial People's Hospital, People's Hospital of Hangzhou Medical College (Hangzhou, China). Samples from 55 patients were collected, including 25 females and 30 males. The age of these patients ranged from 18-80 years old, with an average of 53.8 years. All patients were diagnosed with NPC by pathological examination of biopsy specimens from September 2017 to September 2018 in Zhejiang Provincial People's Hospital, People's Hospital of Hangzhou Medical College. Patients without distant metastasis were classified according to the 7th edition of Union for International Cancer Control Staging System for NPC (15). Biopsy samples of cancer and adjacent tissues were placed in liquid nitrogen immediately, and then stored at $-80^{\circ} \mathrm{C}$ until use. All patients provided written informed consent prior to the initiation of the study.

Cell culture, experimental grouping and transfection. NP69, SUNE1, C666-1, 6-10B and HNE-3 cell lines were obtained from The Cell Bank of Type Culture Collection of Chinese Academy of Sciences (Shanghai, China), and were cultured in RPMI-1640 medium (Thermo Fisher Scientific, Inc., Waltham, MA, USA). The NP69 cell line was used as the control group, which was derived from epithelial cells of the human nasopharynx. Cells were supplied with $10 \%$ fetal bovine serum (HyClone; GE Healthcare Life Sciences, Logan, UT, USA) and cultured at $37^{\circ} \mathrm{C}$ with $5 \% \mathrm{CO}_{2}$. Cells were sub-cultured when cell density reached $80-90 \%$ confluence. The small interfering RNA (siRNA) against FGFR2 (siFGFR2) and negative control (NC)-siRNA were synthesized by Shanghai GenePharma Co., Ltd. (Shanghai, China). The sequences of the FGFR2 siRNA were 5'-CAATAGGACAGTGCTTATT-3' and 5'-CTCTCTATGTCATAGTTGA-3', and the sequence of the NC-siRNA was 5'-AATTCTCCGAACGTGTCACG-3'. SUNE1 and C666-1 cells were transfected with siFGFR2 $(40 \mathrm{nM})$ or NC-siRNA using Lipofectamine ${ }^{\mathrm{TM}} 2000$ transfection reagent (Invitrogen; Thermo Fisher Scientific, Inc.), according to the manufacturer's protocol. After $48 \mathrm{~h}$, the cells were collected and used for subsequent experiments.

To observe the effect of siFGFR2, SUNE1 and C666-1 cells were divided into three groups: Control (blank); NC (transfection with the NC-siRNA); and siFGFR 2 groups (transfection with siFGFR2). Subsequently, to explore the effect of cisplatin, SUNE1 and C666-1 cells were divided into an additional four groups: Control (blank); CIS-1 (treatment with $1 \mu \mathrm{g} / \mathrm{ml}$ cisplatin); CIS-2 (treatment with $10 \mu \mathrm{g} / \mathrm{ml}$ cisplatin); and CIS-3 (treatment with $20 \mu \mathrm{g} / \mathrm{ml}$ cisplatin) groups. Finally, in order to investigate the effect of cisplatin with concomitant FGFR2 downregulation, SUNE1 and C666-1 cells were divided into six groups: Control (blank); $\mathrm{NC}$ (transfection with the NC-siRNA); siFGFR2 (transfection with siFGFR2); CIS (treatment with $10 \mu \mathrm{g} / \mathrm{ml}$ cisplatin); $\mathrm{NC}+\mathrm{CIS}$ (treatment with $10 \mu \mathrm{g} / \mathrm{ml}$ cisplatin and transfection with the NC-siRNA); and siFGFR2+CIS groups (treatment with $10 \mu \mathrm{g} / \mathrm{ml}$ cisplatin and transfection with siFGFR2).

Immunohistochemistry (IHC). FGFR2 expression in tissues of the 55 cases was examined using an immunohistochemical streptomycin avidin-peroxidase (SP) kit (KIT-9706; Fuzhou Maxim Biotech, Co., Ltd., Fuzhou, China). Tissues were fixed with $4 \%$ paraformaldehyde for $24 \mathrm{~h}$ at room temperature (RT), embedded in paraffin and then cut into $4 \mu \mathrm{m}$ sections. The paraffin sections were incubated at $60^{\circ} \mathrm{C}$ for $2 \mathrm{~h}$, treated with xylene at RT for $20 \mathrm{~min}$, and then immersed in $100 \%$ ethanol for $2 \mathrm{~min}, 95 \%$ ethanol for $2 \mathrm{~min}, 90 \%$ ethanol for $2 \mathrm{~min}, 80 \%$ ethanol for $2 \mathrm{~min}, 70 \%$ ethanol for $2 \mathrm{~min}$, and then placed in distilled water. The sections were placed in an incubator containing citrate antigen repair solution (DAS-0010; Fuzhou Maxim Biotech, Co., Ltd.), and were then placed in an autoclave $\left(126^{\circ} \mathrm{C}\right)$ to be steamed for $2 \mathrm{~min}$. Avidin $(25 \mu \mathrm{g} / \mathrm{ml})$ was added to the sections and incubated for $10 \mathrm{~min}$ at RT. Following this, sections were washed 3 times for 5 min with PBS. D-biotin solution was added at RT for $10 \mathrm{~min}$. Sections were washed with PBS 3 times for $5 \mathrm{~min}$ again. An endogenous peroxidase blocker (3\% hydrogen peroxide) was added to the sections and incubated at RT for $15 \mathrm{~min}$, and then washed 3 times for $3 \mathrm{~min}$. Goat serum (10\%; Abcam, Cambridge, MA, USA) was used to block the sections, and sections were incubated for $10 \mathrm{~min}$ at RT. An anti-FGFR2 antibody (cat. no. ab10648; Abcam) was diluted to 1:1,000, and incubated with the sections overnight at $4^{\circ} \mathrm{C}$. Following washing with PBS 3 times for $5 \mathrm{~min}$, a biotin-labeled goat anti-rabbit IgG secondary antibody (cat. no. ab205718; 1:2,000; Abcam) was added to sections for 10 min at RT. Sections were then washed with PBS 3 times for $3 \mathrm{~min}$, and the SP solution was added and the mixture was incubated at RT for $10 \mathrm{~min}$. Sections were then rinsed with PBS 3 times for 3 min and fresh 3,3'-diaminobenzidine solution was added. Sections were incubated for 5-10 min until a color change was observed, and then were rinsed in distilled water immediately. The slides were counterstained with $0.5 \%$ hematoxylin for $3 \mathrm{~min}$ at RT, and then washed. The sections were immersed in $70 \%$ ethanol for $2 \mathrm{~min}, 80 \%$ ethanol for 
$2 \mathrm{~min}, 90 \%$ ethanol for $2 \mathrm{~min}, 100 \%$ ethanol for $2 \mathrm{~min}$, and then treated with $100 \%$ xylene for $2 \mathrm{~min}$, and then observed under a light microscope (magnification, x100 and x200).

The positive proportions of tissue were determined according to the following classification: 0 , no staining; $1,<33 \%$ cell staining; $2,33-66 \%$ cell staining; $3,>66 \%$ cell staining. The staining intensity was then determined according to the following classification: 0 , without staining; 1, light yellow; 2 , brownish yellow; 3 , dark brown. The final classification scores were calculated by combining the 2 scores: 0 , negative; 2-3, weak positive $(+)$; 4, positive $(++)$; and 5-6, positive $(+++)$.

Survival curves of FGFR2. Complete clinical and follow-up records from the 55 NPC cases were gathered. According to the IHC results of FGFR2 expression, the cases with final classification scores $\geq 4$ were grouped as the high FGFR2 expression cases, and the cases with final classification scores $<4$ were grouped as the low FGFR2 expression cases. The overall survival curves of the two groups were then analyzed.

Cell viability assay. Cell vitality of SUNE1 and C666-1 cells was examined using a Cell Counting Kit-8 (CCK-8) (Sigma-Aldrich; Merck KGaA, Darmstadt, Germany). Procedures were performed according the manufacturer's protocols. The cells were then incubated at $37^{\circ} \mathrm{C}$ for $1 \mathrm{~h}$, and the optical density (OD) was measured at $450 \mathrm{~nm}$ using a Multiskan $^{\mathrm{TM}}$ microplate reader (Thermo Fisher Scientific, Inc.). The assays were performed subsequent to culturing for 24 and 48 h post-transfection.

Assessment of cell cycle rates. Cell cycle rates were measured by Vybrant ${ }^{\mathrm{TM}}$ DyeCycle ${ }^{\mathrm{TM}}$ Violet Stain reagent (Thermo Fisher Scientific, Inc.). Briefly, flow cytometry tubes each containing $1 \mathrm{ml}$ cell suspension in complete media at a concentration of $1 \times 10^{6}$ cells/ml was prepared. Then, $1 \mu \mathrm{l}$ Vybrant DyeCycle ${ }^{\mathrm{TM}}$ Violet stain was added to each tube and mixed well. The stain concentration was adjusted to $5 \mu \mathrm{M}$. Cells were incubated at $37^{\circ} \mathrm{C}$ for $30 \mathrm{~min}$ in the dark. Cells were maintained at $37^{\circ} \mathrm{C}$ until analysis. Cells were analyzed without washing or fixing on a flow cytometer at excitation and emission wavelengths of 405 and $440 \mathrm{~nm}$, respectively.

Determination of mRNA levels by reverse transcription quantitative polymerase chain reaction $(R T-q P C R)$. Total RNA was extracted by TRIzol ${ }^{\circledR}$ (Thermo Fisher Scientific, Inc.) from SUNE1 and C666-1 cells and cDNA was synthesized using an iScript ${ }^{\mathrm{TM}}$ cDNA Synthesis kit (Bio-Rad Laboratories, Inc., Hercules, CA, USA). In brief, $1 \mu \mathrm{g}$ RNA of each sample was transcribed into cDNA following the manufacturer's protocol. A Fast Start Universal SYBR-Green Master kit Roche Diagnostics GmbH (Mannheim, Germany) was used to perform the qPCR. The primers used are listed in Table I. The reaction system was set as the following: $12.5 \mu \mathrm{l} 2 \mathrm{X}$ SYBR-Green master mix; $2 \mu \mathrm{l}$ cDNA template; $1 \mu \mathrm{l}$ forward

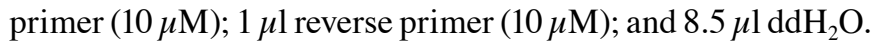
The PCR thermocycling conditions were set as follows: Initial denaturation at $95^{\circ} \mathrm{C}$ for $10 \mathrm{~min}$, followed by 40 cycles of three-step PCR (denaturation at $95^{\circ} \mathrm{C}$ for $15 \mathrm{sec}$, annealing at $60^{\circ} \mathrm{C}$ for $1 \mathrm{~min}$, elongation at $72^{\circ} \mathrm{C}$ for $3 \mathrm{~min}$ ), and final extension at $75^{\circ} \mathrm{C}$ for $10 \mathrm{~min}$. A CFX96 Touch $^{\mathrm{TM}}$ (cat. no. 6093;
Table I. Primers used in reverse transcription-quantitative PCR assay.

\begin{tabular}{ll} 
Gene name & \multicolumn{1}{c}{ Primer sequence (5'-3') } \\
\hline FGFR2 & (F) TTAGAGCCAGAAGAGCCACC \\
Bcl-2 & (R) TACAAGCATAGAGGCCGGAG \\
& (F) TTGAGGAAGTGAACATTTCGGTG \\
Bax & (R) AGGTTCTGCGGACTTAGGTC \\
& (F) GCGAGTGTCTCAAGCGCATC \\
Cyclin D1 & (R) CCAGTTGAAGTTGCCGTCAGAA \\
& (F) CCCTCGGTGTCCTACTTCAA \\
CDC25A & (R) CTTAGAGGCCACGAACATGC \\
(F-actin & (F) CTGTTTGACTCCCCTTCCCT \\
& (F) CACCATTGGCAATGAGCGGTTC \\
& (R) AGGTCTTTGCGGATGTCCACGT
\end{tabular}

FGFR2, fibroblast growth factor receptor 2; Bcl-2, B-cell lymphoma 2; $\mathrm{Bax}, \mathrm{Bcl}-2$-associated $\mathrm{X}$ protein; $\mathrm{CDC} 25 \mathrm{~A}$, cell division cycle gene $25 \mathrm{~A}$; F, forward, R, reverse.

Bio-Rad Laboratories, Inc.) machine was used to conduct the PCR assay. Relative mRNA levels of samples were calculated using the $2^{-\Delta \Delta \mathrm{Cq}}$ method (16).

Extraction and measurement of total protein content and western blot analysis. SUNE1 and C666-1 cells were collected and washed with PBS, and then lysed by radioimmunoprecipitation assay lysis buffer (Beyotime Institute of Biotechnology) according to the manufacturer's protocol. The supernatant of cells was then collected following centrifugation at $4{ }^{\circ} \mathrm{C}$ and $6,000 \mathrm{x}$ g for $15 \mathrm{~min}$. The density of total proteins was measured by the Pierce ${ }^{\mathrm{TM}}$ BCA Protein Assay kit (Thermo Fisher Scientific, Inc.). Briefly, $2 \mu \mathrm{l}$ samples and standard reference proteins, which were diluted to $1,0.5,0.25,0.125$ and $0.0625 \mathrm{~g} / \mathrm{ml}$, respectively, were added to a 96-well plate. $\mathrm{BCA}$ reagent was added to the plate and cells were incubated at $37^{\circ} \mathrm{C}$ for $30 \mathrm{~min}$. The standard curve was generated and the density of the total protein was calculated according to the OD measured at $562 \mathrm{~nm}$ using a Multiskan ${ }^{\mathrm{TM}}$ microplate reader (Thermo Fisher Scientific, Inc.). Then, $20 \mu \mathrm{g}$ total protein of each sample was denatured at $95^{\circ} \mathrm{C}$ for $10 \mathrm{~min}$ and separated by $10 \%$ SDS-PAGE electrophoresis at $100 \mathrm{~V}$ for $2 \mathrm{~h}$. Proteins were transferred onto a PVDF membrane by wet transferring at $90 \mathrm{~V}$ for $2 \mathrm{~h}$, and then blocked with $5 \%$ non-fat milk for $1 \mathrm{~h}$ at RT. Anti-FGFR2 (cat. no. ab10648; Abcam; dilution, 1:1,000), anti-cleaved caspase-3 (Asp175; cat. no. 9664; Cell Signaling Technologies, Danvers, MA, USA; dilution, 1:1,000), anti-B-cell lymphoma 2 (Bcl-2)-associated $\mathrm{X}$ protein (Bax; cat. no. ab32503; Abcam; dilution, 1:1,000), anti-Bcl-2 (cat. no. ab32124; Abcam; dilution, 1:1,000) and anti- $\beta$-actin (cat. no. ab8227; Abcam; dilution, 1:1,000) primary antibodies were added onto the membrane separately. The membrane was then placed on a shaker at $4^{\circ} \mathrm{C}$ overnight. A horseradish peroxidase-conjugated $\operatorname{IgG}(\mathrm{H} \& \mathrm{~L})$ secondary antibody (cat. no. ab6721; Abcam; dilution, 1:2,000) was added 
A

A

\begin{tabular}{lll} 
& Adjacent tissues & \\
\hline 1 & 2 & 3
\end{tabular}

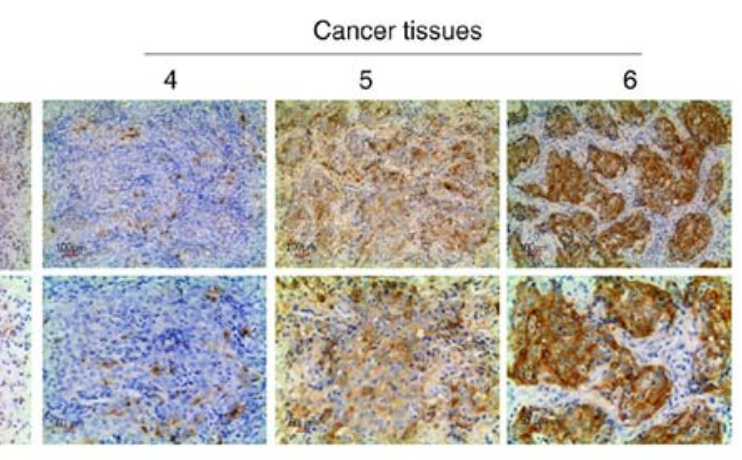

B
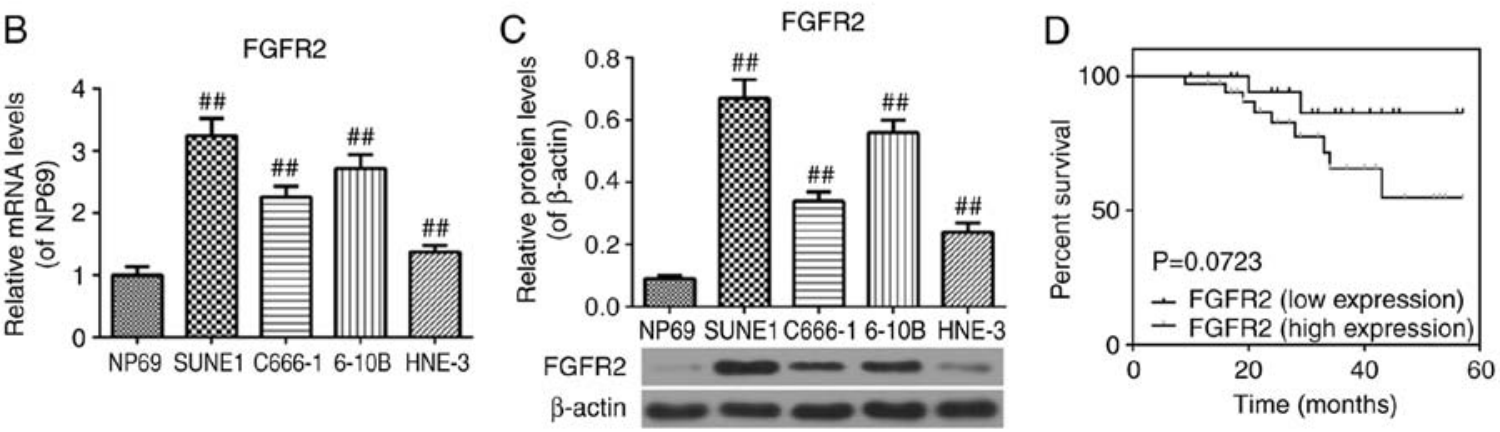

Figure 1. Expression of FGFR2 in tissues from patients with NPC and in NPC SUNE1, C666-1, 6-10B and HNE-3 cell lines. (A) Microscopy images demonstrating FGFR2 expression in cancer and normal adjacent tissues, indicating the different levels of positive staining by immunohistochemistry. (B) Relative mRNA and (C) protein expression levels of FGFR2 in SUNE1, C666-1, 6-10B and HNE-3 cell lines. (D) Survival curves of NPC patients with low or high FGFR2 expression. Data are presented as mean \pm standard deviation. ${ }^{\# \#} \mathrm{P}<0.01$ vs. NP69 cell line. NPC, nasopharyngeal carcinoma; FGFR2, fibroblast growth factor receptor 2 .

following washing of the membrane 3 times with PBS/0.05\% Tween-20 (PBST; Beijing Solarbio Science \& Technology, Co., Ltd., Beijing, China) for $5 \mathrm{~min}$. The membrane was then incubated for $1 \mathrm{~h}$ at RT and washed 3 times in PBST. Protein expression levels were detected by Pierce ${ }^{\mathrm{TM}}$ ECL and a western blot analysis substrate (Thermo Fisher Scientific, Inc.). The densitometric analysis was performed by ImageJ software (v.1.46; National Institutes of Health, Bethesda, MD, USA).

Statistical analysis. GraphPad Prism v.7.0 software (GraphPad Software, Inc., La Jolla, CA, USA was used for statistical analysis. Data was presented as the mean \pm standard deviation. Differences were performed using one-way analysis of variance test followed by Tukey's post-hoc test. Survival analysis was performed using the Kaplan-Meier method, and the Breslow test was used to compare survival curves. $\mathrm{P}<0.05$ was considered to indicate a statistically significant difference.

\section{Results}

FGFR2 is overexpressed in cancer tissues from patients with $N P C$, and in SUNE1, C666-1, 6-10B and HNE-3 cell lines. In order to identify whether FGFR2 was overexpressed in NPC, the expression of FGFR2 in adjacent and cancer tissues from patients with NPC was measured by immunohistochemistry and in NPC SUNE1, C666-1, 6-10B, HNE-3 cell lines using RT-qPCR and western blot analysis. Representative images of each level are demonstrated at magnification x100 and x200 in Fig. 1A. Adjacent tissues exhibited decreased scores $(<3)$ compared with the cancer tissues ( $>4$; Fig. 1A), and the expression of FGFR2 was increased in the SUNE1, C666-1, 6-10B and HNE-3 cell lines compared with the NP69 cell line
$(\mathrm{P}<0.01$; Fig. 1B and $\mathrm{C})$. These data indicated that FGFR2 was overexpressed in NPC.

Overexpression of FGFR2 leads to unfavorable prognoses in patients with NPC. To determine whether the expression of FGFR2 affected the prognosis of patients with NPC, the survival curves of patients with NPC with low and high FGFR2 expression levels were analyzed. According to the Kaplan-Meier analysis, the difference between these groups was not statistically significant $(\mathrm{P}=0.0723)$; however, these data provide evidence to suggest that changes in the expression of FGFR2 may affect the prognosis of patients with NPC to a certain extent $(\mathrm{P}=0.0723$; Fig. 1D). However, additional studies are required to confirm this hypothesis.

FGFR2 silencing decreases the cell viability of SUNE1 and C666-1 cell lines. To investigate whether the cell viability was affected due to the downregulation of FGFR2, cell viability was measured using a CCK-8 assay, and the expression of FGFR2 was examined using RT-qPCR and western blot analysis, following silencing of the expression of FGFR2 using siFGFR2. The expression of FGFR2 was downregulated successfully and cell viability was decreased at $48 \mathrm{~h}$ after siFGFR 2 transfection compared with the control or NC groups in the SUNE1 cell line (Fig. 2A-C). The results were similar in the C666-1 cell line (Fig. 2D-F), suggesting that the downregulation of FGFR2 may decrease the viability of SUNE1 and C666-1 cell lines.

FGFR2 silencing induces cell cycle arrest. To observe whether the cell cycle was affected by FGFR 2 silencing, changes to the phases of the cell cycle, and the mRNA expression levels of cyclin D1 and cell division cycle gene 25A (CDC25A) were 

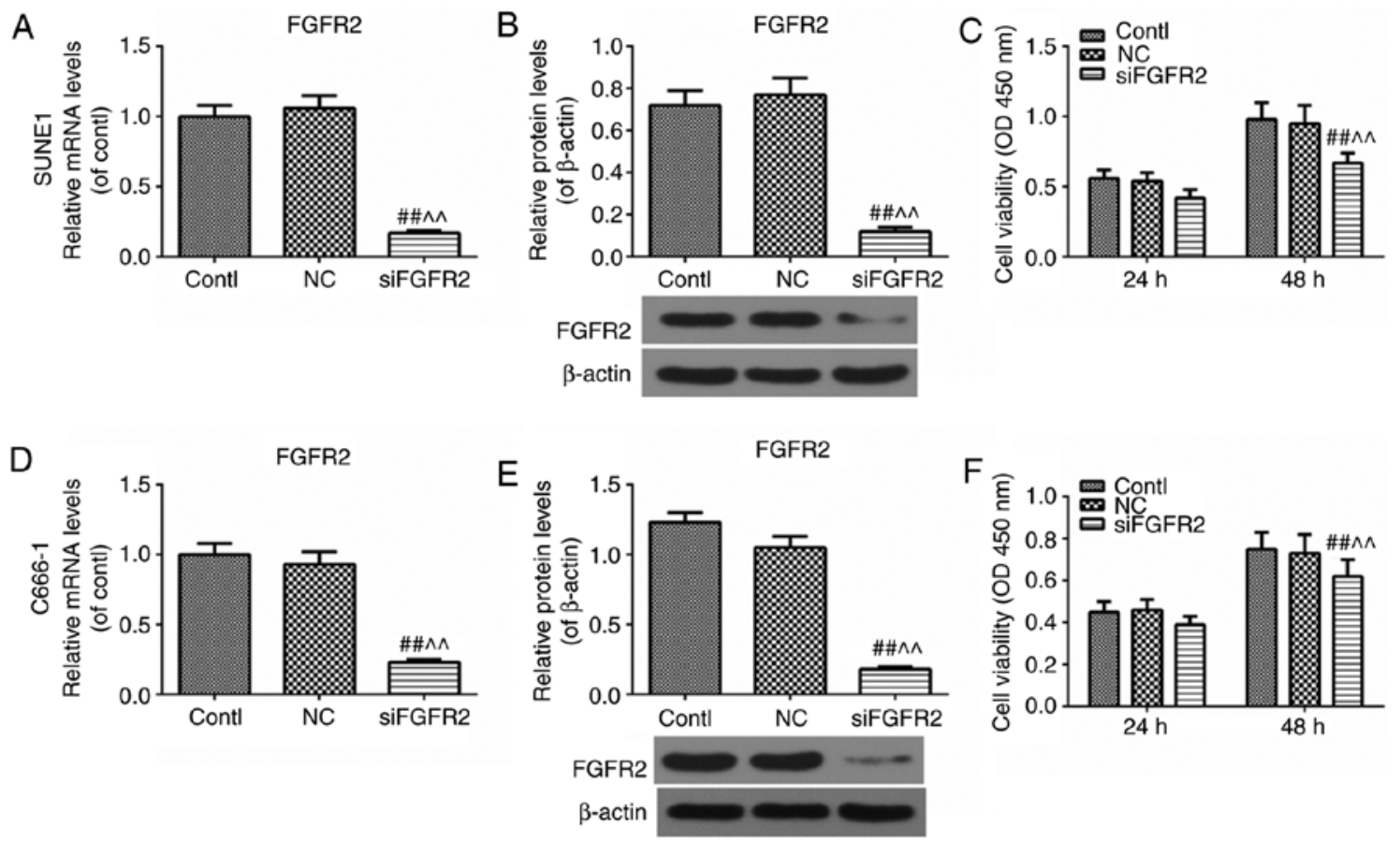

Figure 2. Expression of FGFR2 and the cell viability in control, NC and siFGFR2 groups of SUNE1 and C666-1 cell lines following siFGFR2 transfection. (A) Relative mRNA levels of FGFR2 in the SUNE1 cell line. (B) Relative protein levels of FGFR2 in the SUNE1 cell line. (C) Cell viability in the SUNE1 cell line at 24 and $48 \mathrm{~h}$ after siFGFR2 transfection. (D) Relative mRNA levels of FGFR2 in the C666-1 cell line. (E) Relative protein levels of FGFR2 in the C666-1 cell line. (F) Cell viability in the C666-1 cell line at 24 and $48 \mathrm{~h}$ after siFGFR2 transfection. Data are presented as mean \pm standard deviation. ${ }^{\# \#} \mathrm{P}<0.01$ vs. control group; ${ }^{\wedge} \mathrm{P}<0.01$ vs. NC group. FGFR2, fibroblast growth factor receptor 2; si, small interfering; contl, control; NC, negative control.
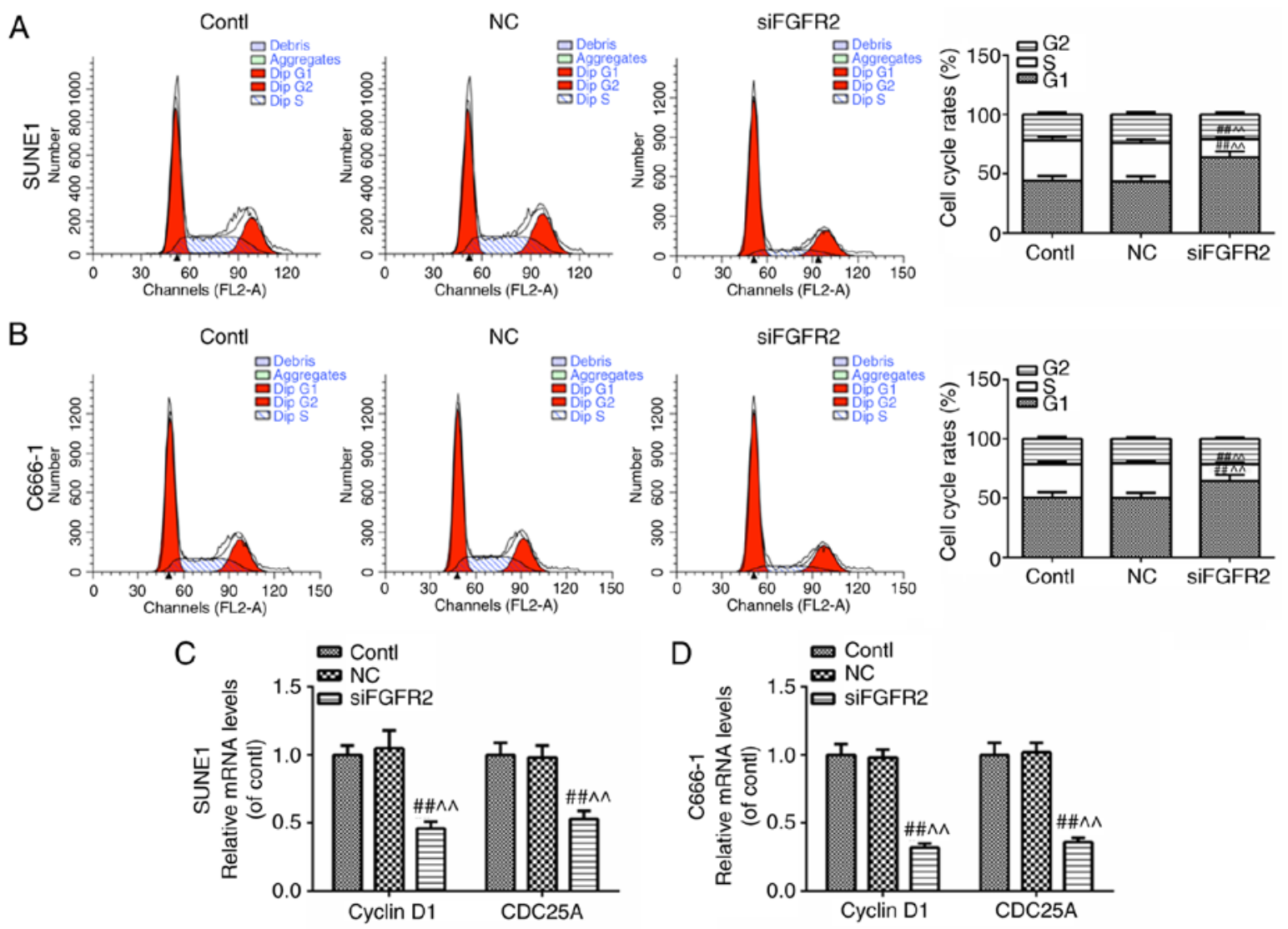

Figure 3. Cell cycle rates and expression levels of cyclin D1 and CDC25A in control, NC and siFGFR2 groups of SUNE1 and C666-1 cell lines following siFGFR2 transfection. (A) The cell cycle rates in the SUNE1 cell line. (B) The cell cycle rates in the C666-1 cell line. (C) Relative mRNA levels of cyclin D1 and CDC25A in the SUNE1 cell line. (D) Relative mRNA levels of cyclin D1 and CDC25A in the C666-1 cell line. Data are presented as mean \pm standard deviation. ${ }^{\# \#} \mathrm{P}<0.01$ vs. control groups; ${ }^{\wedge} \mathrm{P}<0.01$ vs. $\mathrm{NC}$ groups. $\mathrm{CDC} 25 \mathrm{~A}$, cell division cycle gene $25 \mathrm{~A}$; FGFR2, fibroblast growth factor receptor 2 ; si, small interfering; contl, control; NC, negative control. 

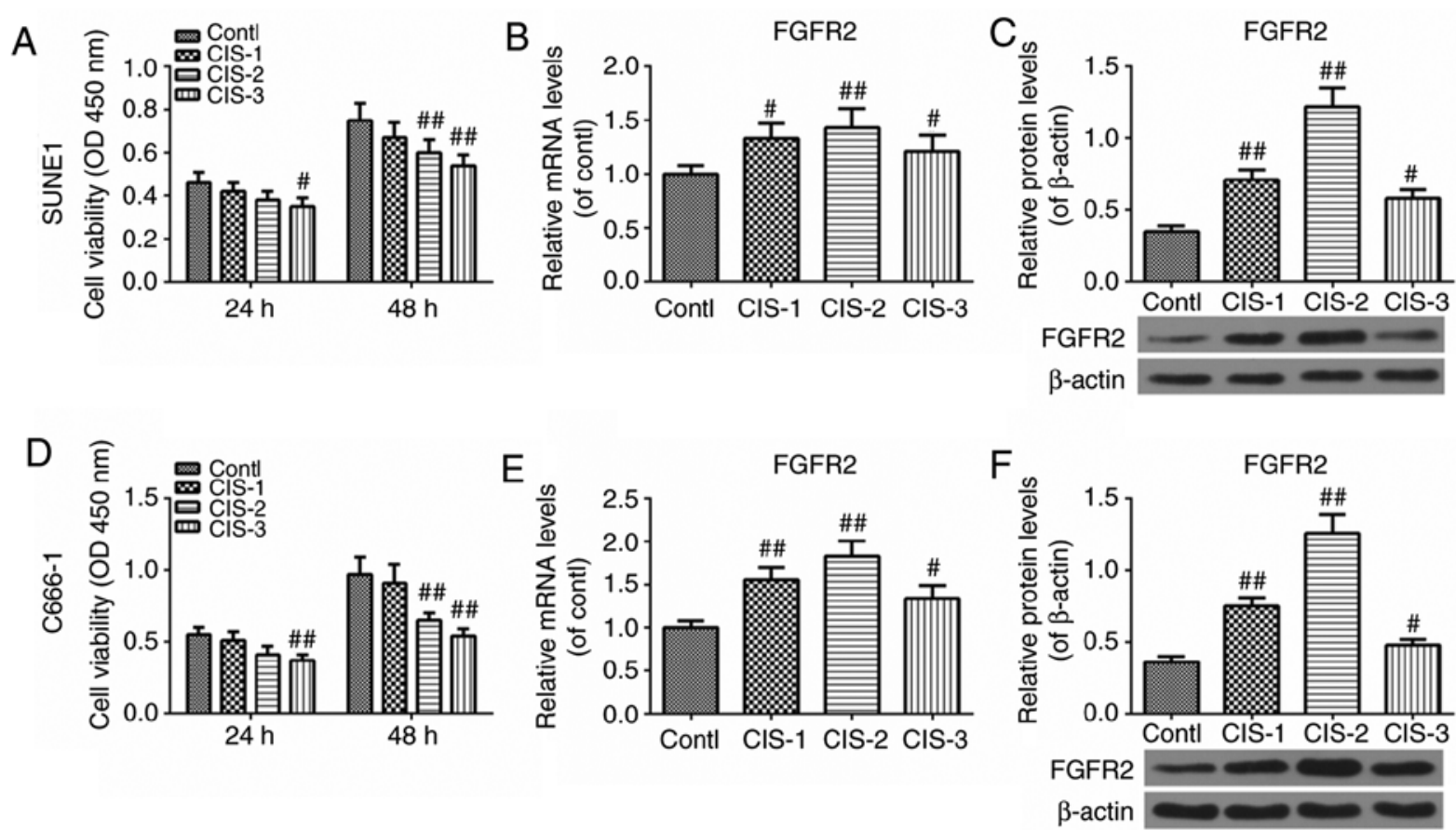

Figure 4. Cell viability and expression of FGFR2 following cisplatin treatment at different concentrations in the control, CIS-1, CIS-2 and CIS-3 groups of SUNE1 and C666-1 cell lines. (A) The cell viability following cisplatin treatment for 24 and $48 \mathrm{~h}$ in the SUNE1 cell line. (B) Relative mRNA levels of FGFR2 in the SUNE1 cell line. (C) Relative protein levels of FGFR2 in the SUNE1 cell line. (D) The cell viability following the treatment of cisplatin for 24 and $48 \mathrm{~h}$ in the C666-1 cell line. (E) Relative mRNA levels of FGFR2 in the C666-1 cell line. (F) Relative protein levels of FGFR2 in the C666-1 cell line. Data are presented as mean \pm standard deviation. ${ }^{* \#} \mathrm{P}<0.01$ and ${ }^{\#} \mathrm{P}<0.01$ vs. control groups. FGFR2, fibroblast growth factor receptor 2; contl, control; CIS, cisplatin; OD, optical density.

investigated by flow cytometry and RT-qPCR, respectively. The G1 phase was prolonged and the S phase was shortened (Fig. 3A and B), and the expression levels of cyclin D1 and CDC25A were decreased in the SUNE1 and C666-1 cell lines (Fig. 3C and D). These data implied that the silencing of FGFR2 may induce cell cycle arrest in the G1 phase.

Cisplatin decreases cell viability and increases FGFR2 expression. Cell viability, FGFR 2 mRNA and FGFR2 protein expression levels following treatment with cisplatin were examined by CCK-8 assay, RT-qPCR and western blot analysis, respectively. As demonstrated in Fig. 4, the cell viability was decreased after $24 \mathrm{~h}$ in the CIS-3 group, and decreased in the CIS- 2 and CIS- 3 groups after $48 \mathrm{~h}$ when compared with the control groups (Fig. 4A). In addition, the expression levels of FGFR2 in the CIS-1, 2, 3 groups were increased compared with the control group; levels were highest in CIS-2 group (Fig. 4B and C). Similar results were observed in the SUNE1 and C666-1 groups (Fig. 4D-F). These results revealed that cisplatin may decrease the viability of NPC cells and increase the expression of FGFR2.

FGFR2 silencing increases the apoptosis induced by cisplatin. To determine whether the overexpression of FGFR2 was involved in the apoptosis effect induced by cisplatin, the apoptosis rate was measured by flow cytometry. As expected, the apoptosis rates in siFGFR2 and CIS groups were increased compared with the control or NC groups (Fig. 5A). Notably, the apoptosis rate in the siFGFR2+CIS group was significantly increased compared with the CIS group (Fig. 5A).
Similar results were observed in the SUNE1 and C666-1 cell lines (Fig. 5B). We hypothesized that the downregulation of FGFR2 may increase the effectiveness of cisplatin-induced apoptosis.

FGFR2 silencing enhances the intrinsic apoptosis pathway induced bycisplatin. To understand the mechanism underlying the improved effect of apoptosis induced by the combination of siFGFR2 and cisplatin, the protein levels of key effector molecules involved in the activation of the intrinsic apoptosis pathway were determined. Significantly, the expression of FGFR2 in the siFGFR2 and siFGFR2+CIS groups was suppressed, but increased in the CIS group compared with the control or NC groups (Fig. 6A). The expression level of cleaved caspase- 3 and Bax were increased in the siFGFR2, CIS, and siFGFR2+CIS groups, with the highest levels observed in siFGFR2+CIS group, compared with the control or NC groups (Fig. 6A). The expression levels of Bcl-2 in the siFGFR2, CIS and siFGFR2+CIS groups were decreased compared with the control or NC groups; notably, the lowest level was observed in the siFGFR2+CIS group (Fig. 6A). The results in the SUNE1 cells were confirmed by similar results in the C666-1 cell line (Fig. 6B). Taken together, these data indicated that the intrinsic apoptosis pathway may be activated by FGFR2 silencing and cisplatin; however, the combination of these two interventions was demonstrated to be more effective compared with each intervention alone, suggesting that the downregulation of FGFR2 may enhance the activation of intrinsic apoptosis pathway induced by cisplatin. 

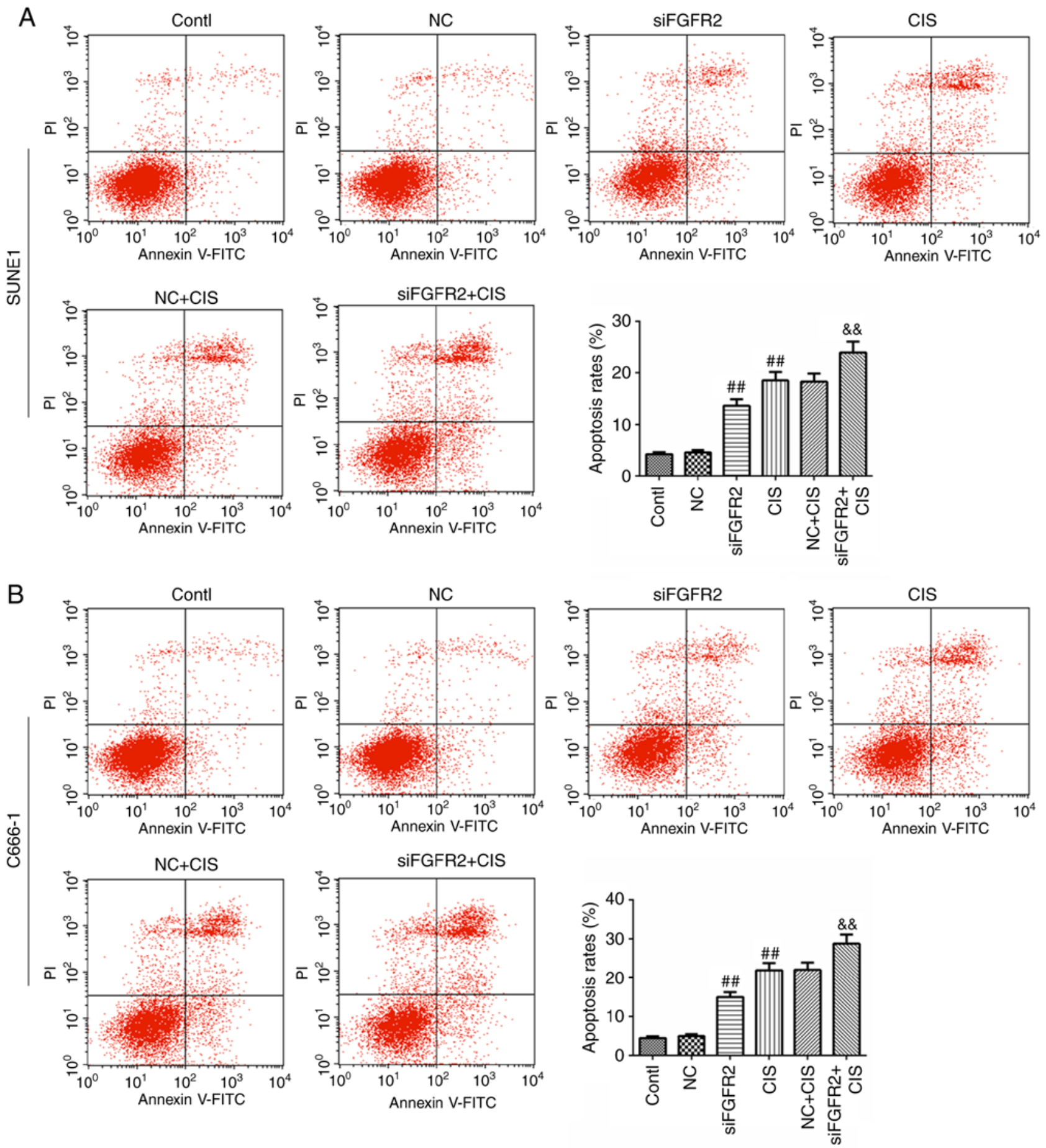

Figure 5. Apoptosis rates in control, NC, siFGFR2, CIS, NC+CIS and siFGFR2+CIS groups of SUNE1 and C666-1 cell lines. (A) The apoptosis rates in the SUNE1 cell line (B) The apoptosis rates in the C666-1 cell line. Data are presented as mean \pm standard deviation. ${ }^{\# \#} \mathrm{P}<0.01 \mathrm{vs.}$ control or NC group; \&\& $\mathrm{P}<0.01$ vs. CIS group. FGFR2, fibroblast growth factor receptor 2; si, small interfering; contl, control; NC, negative control; CIS, cisplatin.

\section{Discussion}

The present study revealed that FGFR2 was overexpressed in cancer tissues from patients with NPC and multiple NPC cell lines, and that the downregulation of FGFR2 decreased the cell viability and induced cell cycle arrest in SUNE1 and C666-1 cell lines. An increased expression of FGFR2 was also observed in cisplatin-treated cells, and the use of cisplatin with siFGFR2 demonstrated increased effectiveness in increasing the apoptosis rate and activating the intrinsic apoptosis pathway of SUNE1 and C666-1 cells compared with the treatment of siFGFR2 or CIS alone, suggesting a potential mechanism for improving the efficacy of cisplatin in the treatment of NPC.

The present study also demonstrated that FGFR2 was highly expressed in the tumor tissues of patients with NPC, and that this level was increased compared with that in adjacent tissues. No significant difference in the outcomes of the survival analyses of patients with low or high expression of FGFR2 was observed; however, the data suggested that changes in the expression of FGFR2 may affect the prognosis 

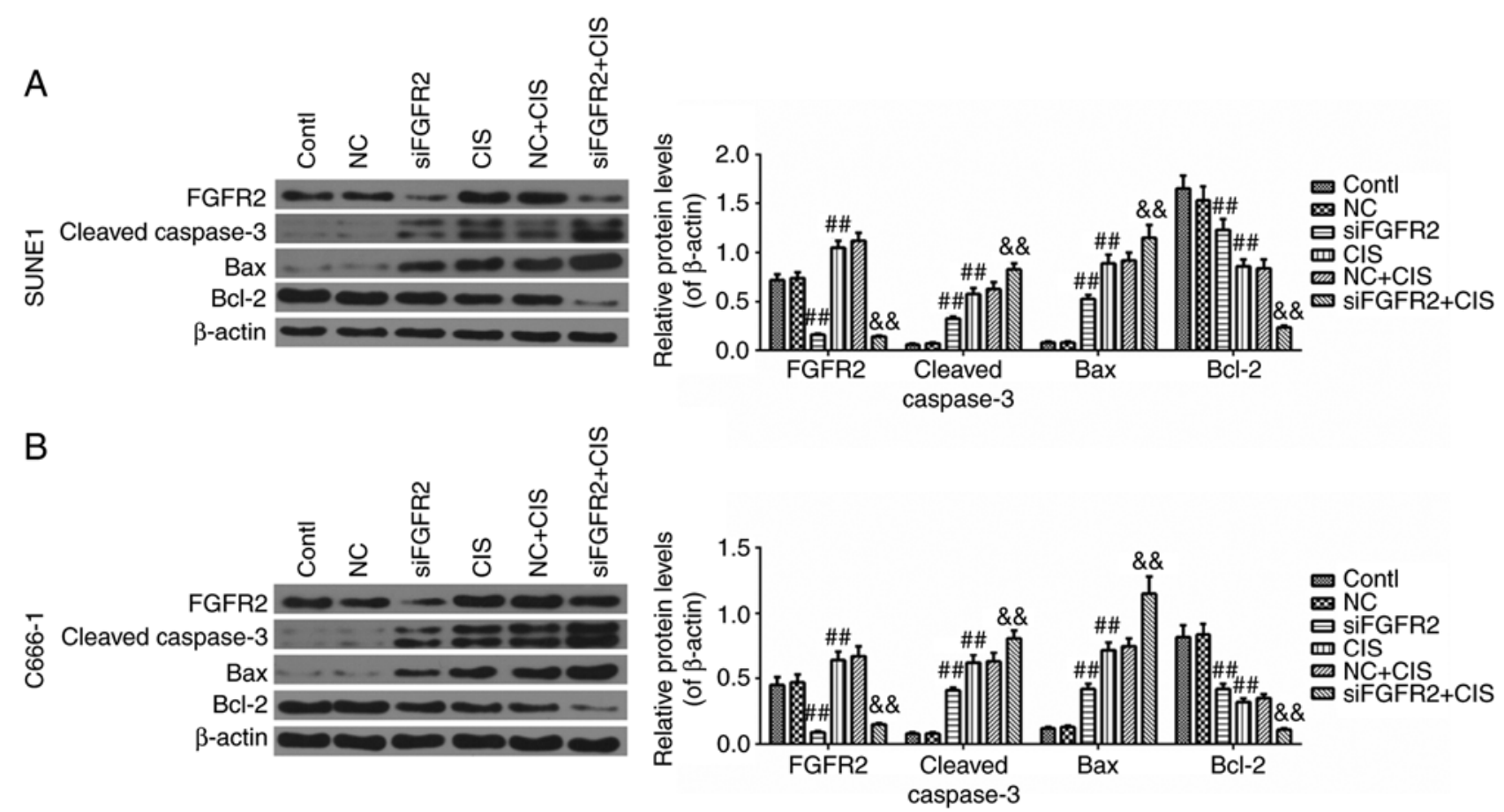

Figure 6. Expression levels of FGFR2, cleaved caspase-3, Bax and Bcl-2 in control, NC, siFGFR2, CIS, NC+CIS and siFGFR2+CIS groups of SUNE1 and C666-1 cell lines. (A) The expression of FGFR2, cleaved Caspase-3, Bax and Bcl-2 in the SUNE1 cell line (B) The expression of FGFR2, cleaved Caspase-3, Bax and $\mathrm{Bcl}-2$ in the C666-1 cell line. Data are presented as mean \pm standard deviation. ${ }^{\# \#} \mathrm{P}<0.01$ vs. control or $\mathrm{NC}$ group; ${ }^{\& \&} \mathrm{P}<0.01$ vs. CIS group. FGFR2, fibroblast growth factor receptor 2; si, small interfering; Bcl-2, B-cell lymphoma 2; Bax, Bcl-2-associated X protein; contl, control; NC, negative control; CIS, cisplatin.

of patients with NPC. To the best of our knowledge, the present study was the first to demonstrate the marked increase in FGFR2 expression in NPC tissues and cell lines. Therefore, FGFR2 may be a promising target in the study of NPC.

To additionally understand the role of FGFR 2 in NPC, the expression of FGFR2 was downregulated, and a decrease in cell viability $48 \mathrm{~h}$ after transfection was observed in the SUNE1 and C666-1 cell lines. Zhang et al (17) demonstrated that FGFR2 was essential for cell proliferation and differentiation in corneal epithelial cells during embryonic development. Zhao et al (18) also revealed that microRNA (miR)-494 was able to inhibit proliferation and promote apoptosis of ovarian cancer cells by targeting FGFR2. Given these data, we hypothesized that the downregulation of FGFR2 may decrease the viability of SUNE1 and C666-1 cell lines.

One of the most basic biological characteristics of malignant tumors is the malignant transformation of cells caused by disruptions in cell cycle regulation and the uncontrolled proliferation of tumor cells. Understanding the expression of cell cycle-associated genes may reveal key effector molecules and biological pathways involved in basic tumor biology (19). During the proliferation of cancer cells in NPC, the acceleration of cell cycle has been demonstrated to be closely associated with cell proliferation (3). Cyclin D1 is a member of the cyclin family that regulates cell cycle progression by binding to protein kinases; abnormalities in its expression may interrupt the cell cycle, which is one of the key mechanisms of cell malignant transformation (20). In eukaryotic cells, the G1 cell cycle checkpoint initiates the cell cycle and promotes cell proliferation, and is considered the primary regulatory point (21). The G1 phase is arrested by the rapid degradation of cyclin D1 protein, if DNA damage is detected in the
G1/S phase (22). CDC25A is the most important member of the cell division cycle gene 25 (CDC25) (23). It has been demonstrated to function in all stages of the cell cycle, and to serve important roles in the cell cycle, mitosis and various physiological activities $(23,24)$. It is also a regulatory factor in cell apoptosis, and the primary regulatory molecule responsible for maintaining DNA damage checkpoint pathways (24). Degradation of CDC25A may cause cell cycle arrest in the G1 or G2 phases, allowing cells to address DNA damage or abnormalities $(25,26)$. A high expression level of CDC25A protein signifies checkpoint dysfunction, causing the cell cycle to continue in the presence of DNA damage, which is a mechanism that is considered to be important in the occurrence and development of malignant tumors $(25,26)$. In addition, the G1 phase is considered to be the phase most sensitive to cisplatin. The present study demonstrated that the proportion of cells in G1 and S phases were increased and decreased, respectively, and that the expression levels of cyclin D1 and CDC25A were decreased following the downregulation of siFGFR2 in SUNE1 and C666-1 cell lines. Lee et al (27), suggested that a single-point mutation in FGFR2 may affect cell cycle in osteoblasts. Yin et al (28), demonstrated that miR-19b-1 may target FGFR2 mRNA and inhibit cell cycle progression from the $S$ phase to the $\mathrm{G} 2 / \mathrm{M}$ phase transition by regulating the expression of cyclin D1. Gredler et al (29), also revealed that FGFR2 regulated cell cycle progression in the urethral and surface ectoderm. These data implied that the downregulation of FGFR2 may induce cell cycle arrest in NPC cells in the G1 phase, inhibiting cell proliferation and increasing the duration of the cell cycle phase that is more sensitive to cisplatin, which may result in an increased efficacy of cisplatin treatment in NPC. 
Notably, the present study identified that increased concentrations (at least $10 \mu \mathrm{g} / \mathrm{ml}$ ) of cisplatin decreased cell viability, and that this decrease in cell viability was observed to be cisplatin concentration-dependent. It was also identified that the FGFR2 mRNA and protein expression levels were increased by cisplatin; the highest levels were exhibited in the $10 \mu \mathrm{g} / \mathrm{ml}$ cisplatin group. Chen et al (30) demonstrated that cisplatin inhibited proliferation and promoted apoptosis in TW03 cells. Huang et al (31) also suggested that cisplatin increased the cell apoptosis and inhibited the cell proliferation of HNE1 cells. Considering these aforementioned data, the results of the present study verified that cisplatin inhibited the viability of NPC cells, and it was hypothesized that the increased expression of FGFR2 was associated with the efficacy of cisplatin treatment in NPC.

Based on the aforementioned data, the present study explored the effects of siFGFR2, cisplatin and the combination of these two interventions on apoptosis. Following the stimulation of intracellular apoptotic factors, Bcl-2 and Bax are activated and bind to the mitochondrial outer membrane, forming pores between the mitochondria and the cytoplasm, which leads to the release of cytochrome $\mathrm{c}$ into the cytoplasm and the activation of caspase- 3 or -7 , thereby initiating the intrinsic apoptosis pathway (32). The results from the present study indicated that the rate of apoptosis was increased following treatment with siFGFR2 and cisplatin; however, the simultaneous treatment of cisplatin with siFGFR2 markedly increased the apoptosis rate of SUNE1 and C666-1 cells compared with the use of siFGFR 2 or cisplatin alone. In addition, the expression of FGFR2 and Bcl-2 were suppressed by the combined treatment of siFGFR 2 and cisplatin, which was more effective compared with the use of siFGFR2 or cisplatin alone in SUNE1 and C666-1 cell lines. In addition, the expression levels of cleaved Caspase-3 and Bax were markedly increased following the combined treatment of siFGFR2 and cisplatin, which was also more effective compared with the use of siFGFR2 or cisplatin alone in SUNE1 and C666-1 cell lines. These results were supported by the data from Cole et al (33), which suggested that the inhibition of FGFR2 increased the rate of apoptosis induced by cisplatin in ovarian cancer. The results from the present study suggested that the downregulation of FGFR2 improved the efficacy of cisplatin treatment on the induction of apoptosis through the activation of intrinsic apoptosis pathway. However, the mechanism by which FGFR2 enhances the sensitivity of NPC cells to cisplatin requires additional study with appropriate in vivo experiments.

In summary, the present study demonstrated that FGFR2 was overexpressed in the cancer tissues of patients with NPC and NPC cell lines, resulting in unfavorable prognoses. The downregulation of FGFR2 decreased cell viability via cell cycle arrest at G1 phase, and increased the efficacy of cisplatin on apoptosis through the intrinsic apoptosis pathway.

\section{Acknowledgements}

Not applicable.

\section{Funding}

No funding was received.

\section{Availability of data and materials}

The datasets used and/or analyzed during the present study are available from the corresponding author on reasonable request.

\section{Authors' contributions}

LP and XK made substantial contributions to the conception and design of the study. LS and LP were responsible for data acquisition, data analysis and interpretation. XK and LS were involved in drafting the article and critically revising it for important intellectual content. All authors read and approved the final manuscript. LP and XK agreed to be accountable for all aspects of the work in ensuring that questions related to the accuracy or integrity of the work are appropriately investigated and resolved.

\section{Ethics approval and consent to participate}

The study protocol was approved by the Ethics Board of Zhejiang Provincial People's Hospital, People's Hospital of Hangzhou Medical College (Hangzhou, China). All procedures involving human participants were performed in accordance with the ethical standards of the institutional and/or national research committee and with the 1964 Helsinki declaration and its later amendments or comparable ethical standards. All patients provided written informed consent.

\section{Patient consent for publication}

Not applicable.

\section{Competing interests}

The authors declare that they have no competing interests.

\section{References}

1. Liu X, Tang LL, Du XJ, Li WF, Chen L, Zhou GQ, Guo R, Liu Q, Sun Y and Ma J: Changes in disease failure risk of nasopharyngeal carcinoma over time: Analysis of 749 patients with long-term follow-up. J Cancer 8: 455-459, 2017.

2. Wilmot VV and Hathorn I: Surgical management of nasal stenosis following chemoradiation for nasopharyngeal carcinoma. J Laryngol Otol 131: 429-432, 2017.

3. Strazzulla A, Barreca GS, Giancotti A, Pisani V, Costa C, Zicca E, La Boria A, Roveda L, Liberto MC, Tucci L, et al: Nasopharyngeal carcinoma: Review of the literature with a focus on therapeutical implications. Infez Med 23: 224-229, 2015.

4. Zhang X, Ibrahimi OA, Olsen SK, Umemori H, Mohammadi M and Ornitz DM: Receptor specificity of the fibroblast growth factor family. The complete mammalian FGF family. J Biol Chem 281: 15694-15700, 2006.

5. Grose R and Dickson C: Fibroblast growth factor signaling in tumorigenesis. Cytokine Growth Factor Rev 16: 179-186, 2005.

6. Katoh M and Katoh M: FGFR2 and WDR11 are neighboring oncogene and tumor suppressor gene on human chromosome 10q26. Int J Oncol 22: 1155-1159, 2003.

7. Turner $\mathrm{N}$ and Grose R: Fibroblast growth factor signalling: From development to cancer. Nat Rev Cancer 10: 116-129, 2010.

8. Jung EJ, Jung EJ, Min SY, Kim MA and Kim WH: Fibroblast growth factor receptor 2 gene amplification status and its clinicopathologic significance in gastric carcinoma. Hum Pathol 43: 1559-1566, 2012.

9. Matsumoto K, Arao T, Hamaguchi T, Shimada Y, Kato K, Oda I, Taniguchi H, Koizumi F, Yanagihara K, Sasaki H, et al: FGFR2 gene amplification and clinicopathological features in gastric cancer. Br J Cancer 106: 727-732, 2012. 
10. Byron SA, Gartside MG, Wellens CL, Goodfellow PJ, Birrer MJ, Campbell IG and Pollock PM: FGFR2 mutations are rare across histologic subtypes of ovarian cancer. Gynecol Oncol 117: $125-129,2010$

11. Riddell IA: Cisplatin and oxaliplatin: Our current understanding of their actions. Met Ions Life Sci 18, 2018.

12. Köberle B, Tomicic MT, Usanova S and Kaina B: Cisplatin resistance: Preclinical findings and clinical implications. Biochim Biophys Acta 1806: 172-182, 2010.

13. Wu P, Tang Y, He J, Qi L, Jiang W and Zhao S: ARC is highly expressed in nasopharyngeal carcinoma and confers X-radiation and cisplatin resistance. Oncol Rep 30: 1807-1813, 2013.

14. Song Y, Zhou X, Bai W and Ma X: FBW7 increases drug sensitivity to cisplatin in human nasopharyngeal carcinoma by downregulating the expression of multidrug resistance-associated protein. Tumour Biol 36: 4197-4202, 2015.

15. Edge SB and Compton CC: The American Joint Committee on Cancer: The 7th edition of the AJCC cancer staging manual and the future of TNM. Ann Surg Oncol 17: 1471-1474, 2010.

16. Livak KJ and Schmittgen TD: Analysis of relative gene expression data using real-time quantitative PCR and the 2(-Delta Delta C(T)) method. Methods 25: 402-408, 2001.

17. Zhang J, Upadhya D, Lu L and Reneker LW: Fibroblast growth factor receptor 2 (FGFR2) is required for corneal epithelial cell proliferation and differentiation during embryonic development. PLoS One 10: e0117089, 2015.

18. Zhao X, Zhou Y, Chen YU and Yu F: miR-494 inhibits ovarian cancer cell proliferation and promotes apoptosis by targeting FGFR2. Oncol Lett 11: 4245-4251, 2016.

19. Williams GH and Stoeber K: The cell cycle and cancer. J Pathol 226: 352-364, 2012.

20. Feldt M, Bjarnadottir O, Kimbung S, Jirström K, Bendahl PO, Veerla S, Grabau D, Hedenfalk I and Borgquist S: Statin-induced anti-proliferative effects via cyclin D1 and p27 in a window-of-opportunity breast cancer trial. J Transl Med 13: 133, 2015.

21. Neumann J, Boerries M, Köhler R, Giaisi M, Krammer PH, Busch H and Li-Weber M: The natural anticancer compound rocaglamide selectively inhibits the G1-S-phase transition in cancer cells through the ATM/ATR-mediated Chk1/2 cell cycle checkpoints. Int J Cancer 134: 1991-2002, 2014.

22. Roque T, Haton C, Etienne O, Chicheportiche A, Rousseau L, Martin L, Mouthon MA and Boussin FD: Lack of a p21waf1/cipdependent G1/S checkpoint in neural stem and progenitor cells after DNA damage in vivo. Stem Cells 30: 537-547, 2012.
23. Boutros R, Lobjois V and Ducommun B: CDC25 phosphatases in cancer cells: Key players? Good targets? Nat Rev Cancer 7: 495-507, 2007.

24. Shen T and Huang S: The role of Cdc25A in the regulation of cell proliferation and apoptosis. Anticancer Agents Med Chem 12: 631-639, 2012.

25. Kang T, Wei Y, Honaker Y, Yamaguchi H, Appella E, Hung MC and Piwnica-Worms H: GSK-3 beta targets Cdc25A for ubiquitin-mediated proteolysis, and GSK-3 beta inactivation correlates with Cdc25A overproduction in human cancers. Cancer Cell 13: 36-47, 2008

26. Sur S and Agrawal DK: Phosphatases and kinases regulating CDC25 activity in the cell cycle: Clinical implications of CDC25 overexpression and potential treatment strategies. Mol Cell Biochem 416: 33-46, 2016.

27. Lee KM, Santos-Ruiz L and Ferretti P: A single-point mutation in FGFR2 affects cell cycle and Tgfbeta signalling in osteoblasts. Biochim Biophys Acta 1802: 347-355, 2010.

28. Yin R, Bao W, Xing Y, Xi T and Gou S: MiR-19b-1 inhibits angiogenesis by blocking cell cycle progression of endothelial cells. Biochem Biophys Res Commun 417: 771-776, 2012.

29. Gredler ML, Seifert AW and Cohn MJ: Tissue-specific roles of Fgfr2 in development of the external genitalia. Development 142: 2203-2212, 2015

30. Chen J, Liu S, Li Q and Peng J: Combination of cytosine arabinoside and cisplatin enhances inhibition of cell proliferation and promotes apoptosis of resistant nasopharyngeal carcinoma cells. Xi Bao Yu Fen Zi Mian Yi Xue Za Zhi 31: 379-382, 386, 2015 (In Chinese).

31. Huang YY,Pu LJ, Song LL, Ma LY, Liu H and Jiang CC: Knockdown of GRP78 enhances cell death by cisplatin and radiotherapy in nasopharyngeal cells. Anticancer Drugs 27: 726-733, 2016.

32. Green DR and Llambi F: Cell death signaling. Cold Spring Harb Perspect Biol 7, 2015.

33. Cole C, Lau S, Backen A, Clamp A, Rushton G, Dive C, Hodgkinson C, McVey R, Kitchener H and Jayson GC: Inhibition of FGFR2 and FGFR1 increases cisplatin sensitivity in ovarian cancer. Cancer Biol Ther 10: 495-504, 2010.

This work is licensed under a Creative Commons Attribution-NonCommercial-NoDerivatives 4.0 International (CC BY-NC-ND 4.0) License. 